\title{
Plasmacytoid dendritic cells in pulmonary lymphoid follicles of patients with COPD
}

\author{
G.R. Van Pottelberge*, K.R. Bracke*, S. Van den Broeck*, S.M. Reinartz ${ }^{\#}$, \\ C.M. van Drunen ${ }^{\#}$, E.F. Wouters ${ }^{\dagger}$, G.M. Verleden ${ }^{+}$, F.E. Vermassen ${ }^{\S}$, \\ G.F. Joos* and G.G. Brusselle*
}

ABSTRACT: Plasmacytoid dendritic cells (pDCs) are professional antigen-presenting cells with antiviral and tolerogenic capabilities. Viral infections and autoimmunity are proposed to be important mechanisms in the pathogenesis of chronic obstructive pulmonary disease (COPD).

The study aimed to quantify blood dendritic cell antigen 2-positive pDCs in lungs of subjects with or without COPD by immunohistochemistry and flow cytometry, combined with the investigation of the influence of cigarette smoke extract (CSE) on the function of pDCs in vitro.

pDCs were mainly located in lymphoid follicles, a finding compatible with their expression of lymphoid homing chemokine receptors CXCR3 and CXCR4. PDC accumulated in the lymphoid follicles and in lung digests of patients with mild to moderate COPD, compared with smokers without airflow limitation and patients with COPD Global Initiative for Chronic Obstructive Lung disease (GOLD) stage III-IV. Exposing maturing PDC of healthy subjects to CSE in vitro revealed an attenuation of the expression of co-stimulatory molecules and impaired interferon- $\alpha$ production. Maturing PDC from patients with COPD produced higher levels of tumour necrosis factor (TNF)- $\alpha$ and interleukin (IL)-8 compared to pDC from healthy subjects.

CSE significantly impairs the antiviral function of PDCs. In COPD, a GOLD stage dependent accumulation of $\mathrm{pDC}$ in lymphoid follicles is present, combined with an enhanced production of TNF- $\alpha$ and IL-8 by maturing pDCs.

KEYWORDS: Airway inflammation, chronic obstructive pulmonary disease, cigarette smoke, dendritic cell maturation, lymphoid follicle, plasmacytoid dendritic cells

hronic obstructive pulmonary disease (COPD) is a chronic inflammatory disease of the airways and lung parenchyma, inducing substantial morbidity and mortality worldwide [1]. The inflammatory process causes narrowing of the small airways (obstructive bronchiolitis), leading to an airflow limitation that is not fully reversible. In addition, there is a destruction of the alveolar parenchyma (emphysema), resulting in impaired gas exchange and reduced elastic recoil of the lung. In Western countries, cigarette smoking is by far the most important risk factor for developing COPD [2]. The exact pathogenetic mechanisms that drive ongoing inflammation despite smoking cessation still remain to be elucidated. Several pathogenetic entities, such as low-grade bacterial and viral infections, autoimmune responses against changed epitopes and genetic predispositions, have been proposed in this respect [3].
The inflammatory process in COPD comprises both the innate immune response (with epithelial activation and infiltration of neutrophils and macrophages) as well as the adaptive immune response (with influx of cytotoxic CD8+ T-cells, CD4+ T-helper cells and B-cells). In addition, increased numbers of lymphoid follicles are found in the lungs of patients with COPD [4, 5].

Dendritic cells (DCs) are professional antigenpresenting cells of haematopoietic origin, linking these innate and adaptive immune responses. Using specific receptors, DCs sense for danger signals while sampling their environment for antigens. DCs process the antigen, present it on major histocompatibility class II and I molecules and integrate this information with the sensed danger signals by upregulating co-stimulatory molecules and producing specific cytokines. DCs then form an immunological synapse by meeting
AFFILIATIONS

*Laboratory for Translational Research in Obstructive Pulmonary Diseases, Dept of Respiratory Medicine,

${ }^{\S}$ Dept of Thoracic and Vascular Surgery, Ghent University Hospital, Ghent, and

${ }^{+}$Dept of Respiratory Medicine, University Hospital Gasthuisberg, Catholic University of Leuven, Leuven, Belgium.

"Dept of Otorhinolaryngology, Academic Medical Center, Amsterdam, and

'Dept of Respiratory Medicine, University Hospital Maastricht, Maastricht, The Netherlands.

CORRESPONDENCE

G.R. Van Pottelberge

Dept of Respiratory Medicine

Ghent University Hospital 7K12 IE

De Pintelaan 185

B9000 Ghent

Belgium

E-mail: geert.vanpottelberge@

ugent.be

Received:

Sept 042009

Accepted after revision:

March 092010

First published online:

March 292010 
naïve lymphocytes, directing the proliferation of antigenspecific T-cells and, thus, orchestrating the adaptive immune response [6].

In general, two major distinct subsets of DCs are known: myeloid dendritic cells (mDCs) and plasmacytoid dendritic cells (pDCs) [7]. pDCs represent a unique population of professional antigen-presenting cells with a plasma cell-like morphology and a unique surface receptor phenotype, capable of producing large amounts of type I interferons in response to viruses and nucleic acid-containing complexes from the host, sensed through Toll-like receptors (TLRs)-7 and -9 [8, 9]. Apart from this innate antiviral defence function, pDCs play a crucial role in maintaining tolerance by expressing the enzyme indoleamine 2,3 dioxygenase which induces T-cell death by depleting the amino acid tryptophan [10]. In addition, through upregulating inducible T-cell co-stimulator ligand, pDCs are able to generate regulatory T-cells [11]. Taken together, evidence suggests that pDCs play an important role in peripheral tolerance and in antiviral defence mechanisms.

We and others described the presence of mDCs and pDCs in human lungs, using flow cytometry on single cell suspensions of digested human lung tissue and in bronchoalveolar lavage (BAL) fluid [12-15]. Recently, more evidence has become available on the different subsets of $\mathrm{mDC}$, their function and role in the pathogenesis of respiratory diseases such as COPD [16-19]. However, until now, the exact location of pDCs in the human lung was unknown. In this study, we were interested in identifying pDCs in the small airways. Moreover, considering the important immunological role of pDCs, we hypothesised that, in smokers and in patients with COPD, pDCs could be altered in number and function, contributing to impaired antiviral defence and/or loss of tolerance, both alleged mechanistic concepts in the pathogenesis of COPD [20].

This study describes for the first time the distribution of pDCs in the small airways of human lungs, highlighting the concentration of pDCs in lymphoid follicles. In addition, there is a significant accumulation of pDCs in lymphoid follicles of patients with mild to moderate COPD compared with smokers without airflow limitation. Finally, we found an important impact of cigarette smoke extract (CSE) in vitro on the innate and adaptive functions of pDC.

\section{MATERIALS AND METHODS \\ Lung tissue}

Tissue was obtained from surgical lung resection specimens of patients diagnosed with solitary pulmonary lesions at the Ghent University Hospital, Ghent, Belgium. Lung tissue at maximum distance from the pulmonary lesion, and without signs of retro-obstructive pneumonia or tumour invasion, was collected by a pathologist. None of the patients operated for malignancy were treated with neo-adjuvant chemotherapy. Lung tissue from end-stage COPD was obtained from explant lungs from patients undergoing lung transplantation (University Hospital Gasthuisberg, Leuven, Belgium) or lung volume reduction surgery (Maastricht University Medical Centre, Maastricht, the Netherlands). All patients signed informed consent prior to surgery and were interviewed about their smoking habits and medication use. COPD diagnosis and severity was defined using pre-operative spirometry according to the Global Initiative for Chronic Obstruction Lung Disease (GOLD) classification [2]. This study was approved by the medical ethical committee of the Ghent University Hospital, University Hospital Gasthuisberg Leuven and the Maastricht University Medical Centre.

\section{Histology}

Cryosections were incubated with anti-blood dendritic cell antigen 2 (BDCA-2; CD303) monoclonal antibody (clone AC141, Miltenyi Biotec, Bergisch Gladbach, Germany). Langerin (CD207) immunohistochemical staining was performed as described previously [16]. Details on the immunohistochemical stainings are provided in the online supplementary material.

\section{Image analysis}

pDCs in small airways were quantified using a computerised image analysis system (Axiocam, Axioskop II mot + KS400; Zeiss, Oberkochen, Germany). Airways without cartilage that had a perimeter of the basement membrane $<6,000 \mu \mathrm{m}$ were selected for analysis [21]. Lymphoid follicles near small airways were identified in haematoxylin-stained sections at $100 \times$ magnification as an aggregate of contiguous mononuclear cells [22]. In adjacent sections, follicles with a B-cell zone were identified by CD3/CD20 double staining. Follicle boundaries were delineated by tracing the perimeter and the area was calculated using Image J software (National Institutes of Health, Bethesda, MD, USA). The number of BDCA-2positive cells was counted within these follicles. The observers (G.R. Van Pottelberge and S. Van den Broeck) were blinded for clinical data. More information is available in the online supplementary material.

\section{Flow cytometry}

Resection specimens were processed as described previously to obtain single cell suspensions of pulmonary mononuclear cells [12]. Monoclonal antibodies and equipment used are presented in the online supplementary material.

\section{In vitro $p D C$ culture}

CSE was prepared as described previously [23]. pDCs were isolated from fresh blood of healthy nonsmoking volunteers and of patients with COPD. All patients with COPD were exsmokers and had a spirometry compatible with GOLD stage II. None of the patients used systemic corticosteroids. Subjects were free from exacerbation or infection during the 2 months prior to the study. All participants signed informed consent prior to the study. pDCs were isolated by negative selection using Plasmacytoid Dendritic Cell Isolation Kit MicroBeads (Miltenyi Biotec).

pDCs were incubated with $3 \mu \mathrm{g} \cdot \mathrm{mL}^{-1} \mathrm{CpG}$-oligodeoxynucleotides (2216 CpG-type A) or $10 \mu \mathrm{g} \cdot \mathrm{mL}^{-1}$ imiquimod-R837 (Invivogen, San Diego, CA, USA) in the presence or absence of CSE for $18 \mathrm{~h}$ at $37^{\circ} \mathrm{C}$ under a humidified atmosphere with $5 \% \mathrm{CO}_{2}$.

Interferon- $\alpha$ concentration in the supernatant was measured using a sandwich ELISA (PBL Interferon Source, Piscataway, NJ, USA). Other cytokines were measured using cytometric bead array human inflammation kit. The expression of maturation markers by pDC was analysed by flow cytometry. Antibodies used are described in the online supplementary material. 


\section{Statistical analysis}

For the immunohistochemical study, statistical analysis was carried out in SPSS 16.0 (SPSS Inc. Chicago, IL, USA). When evaluating differences in continuous variables between multiple independent groups, the Kruskal-Wallis test was used. Where values of probability were $<0.05$, selected pairs of groups were investigated by the Mann-Whitney U-test. Spearman rank test was used to examine correlations. p-values $<0.05$ were considered significant. For the in vitro study, statistical analysis was carried out in Graph Pad Prism (Graph Pad software Inc. San Diego, CA, USA). Relative expression of maturation markers was analysed using the one sample t-test. Other comparisons were investigated by the paired and unpaired t-test.

\section{RESULTS}

Identification of pDCs in small airways of human lungs

Using immunohistochemical staining for the specific marker BDCA-2, pDCs were detected at low numbers in the small airways of human lungs (fig. 1). Appropriate isotype control staining was performed on human airways and tonsils in order to assure specificity (shown in figure E1 in the online supplementary material). pDCs were mainly found in the adventitia of the small airways and to a limited extent in the lamina propria and the epithelium. Interestingly, pDCs in small airways were often located in lymphoid follicles (fig. 1b). The localisation of the pDCs in these lymphoid follicles was examined by CD3/CD20 double staining in adjacent sections (figure E2 in the online supplementary material). Quantification revealed that mean $\pm S D 72.1 \pm 33.1 \%$ of the $\mathrm{pDCs}$ in lymphoid follicles were located in the T- cell zone.

\section{Pulmonary pDCs express CXCR3 and CXCR4}

In order to investigate the lymphoid homing potential of pulmonary pDCs, the expression of lymphoid homing chemokine receptors CXCR3, CXCR4 and CCR7 was assessed by flow cytometry on labelled single cell suspensions of digested human lung tissue (fig. 2) using the previously described gating strategy [12]. Pulmonary pDCs showed high expression of CXCR3 and CXCR4. Importantly, the expression of CCR7 was very low in these pDCs. When comparing the expression of these chemokine receptors between smokers without airflow limitation $(n=3)$ and patients with $\operatorname{COPD}(n=2)$, a trend towards higher expression of CXCR3 was observed in patients with COPD (fig. 2f).

\section{Quantification of pDCs in small airways in patients with COPD and subjects without COPD}

The characteristics of the study population are shown in table 1. A total number of 74 subjects was investigated. The population consisted of never smokers $(n=10)$, smokers without COPD $(n=22)$, COPD GOLD stage I-II subjects $(n=28)$ and COPD GOLD stage III-IV subjects $(n=14)$. Patients with mild to moderate COPD did not use inhaled or systemic corticosteroids.

Quantification of the number of pDCs in the total airway wall, epithelium, lamina propria or adventitia of the small airways, excluding areas with lymphoid follicles, revealed no significant differences between the study groups (fig. 3a and figure $\mathrm{E} 3$ in the online supplementary material). In addition, there were no differences between current and ex-smoking subjects, both with or without airflow limitation (data not shown).
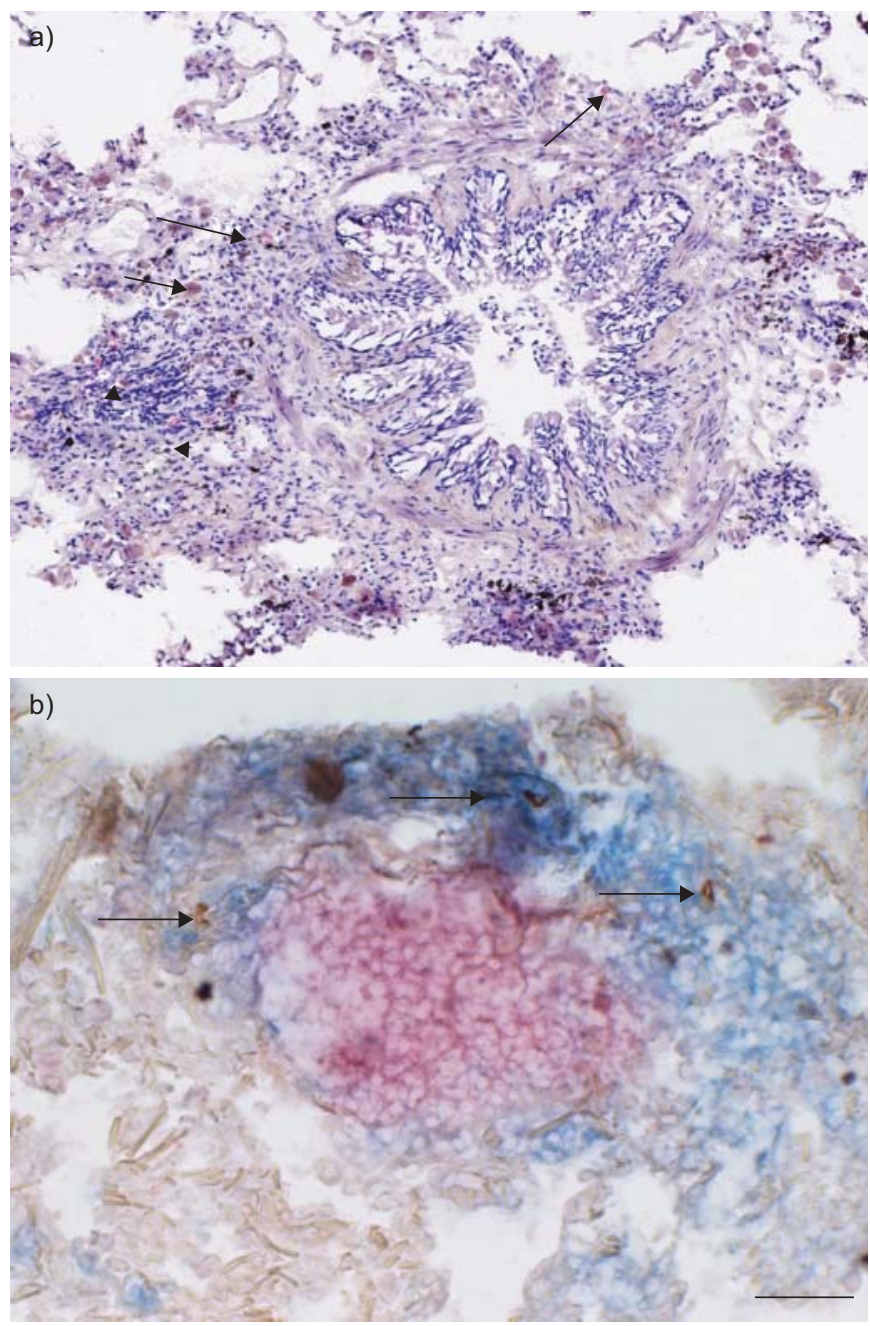

FIGURE 1. Identification of plasmacytoid dendritic cells (pDCs) in small airways. a) Cryosection of human lung (smoker without airflow limitation) was incubated with anti-BDCA-2 antibody, stained with new fuchsin (red colour) and counterstained with haematoxylin. pDCs are located in the adventitia (black arrows) and concentrated in the lymphoid follicle (arrowheads) (original magnification $100 \times$ ). b) Triple staining of a cryosection of human lung tissue for CD3 (blue colour indicates the T-cell zone), CD20 (red-pink colour indicates the B-cell zone) and BDCA-2 (brown colour indicates pDCs). pDCs (arrows) are mainly found in the T-cell zone of lymphoid follicles. Scale bar $=50 \mu \mathrm{M}$.

There were no significant correlations between the number of pDCs and the number of langerin-positive mDCs in the total airway wall. However, when subdividing the subjects into the different study groups according to GOLD stage, a significant positive correlation between the number of pDCs and langerinpositive $\mathrm{mDCs}$ in the total airway wall emerged $\left(r_{\mathrm{s}}=0.64\right.$, $\mathrm{p}=0.004)$ in patients with COPD GOLD stage I. Interestingly, this positive correlation was lost in patients with COPD GOLD stage III-IV (figure E4 in the online supplementary material).

\section{Quantification of pDCs in lymphoid follicles}

In the same study population, small airways with lymphoid follicles were identified. The percentage of airways with a lymphoid follicle was significantly higher in COPD GOLD stage III-IV compared to the COPD GOLD stage I-II subjects 

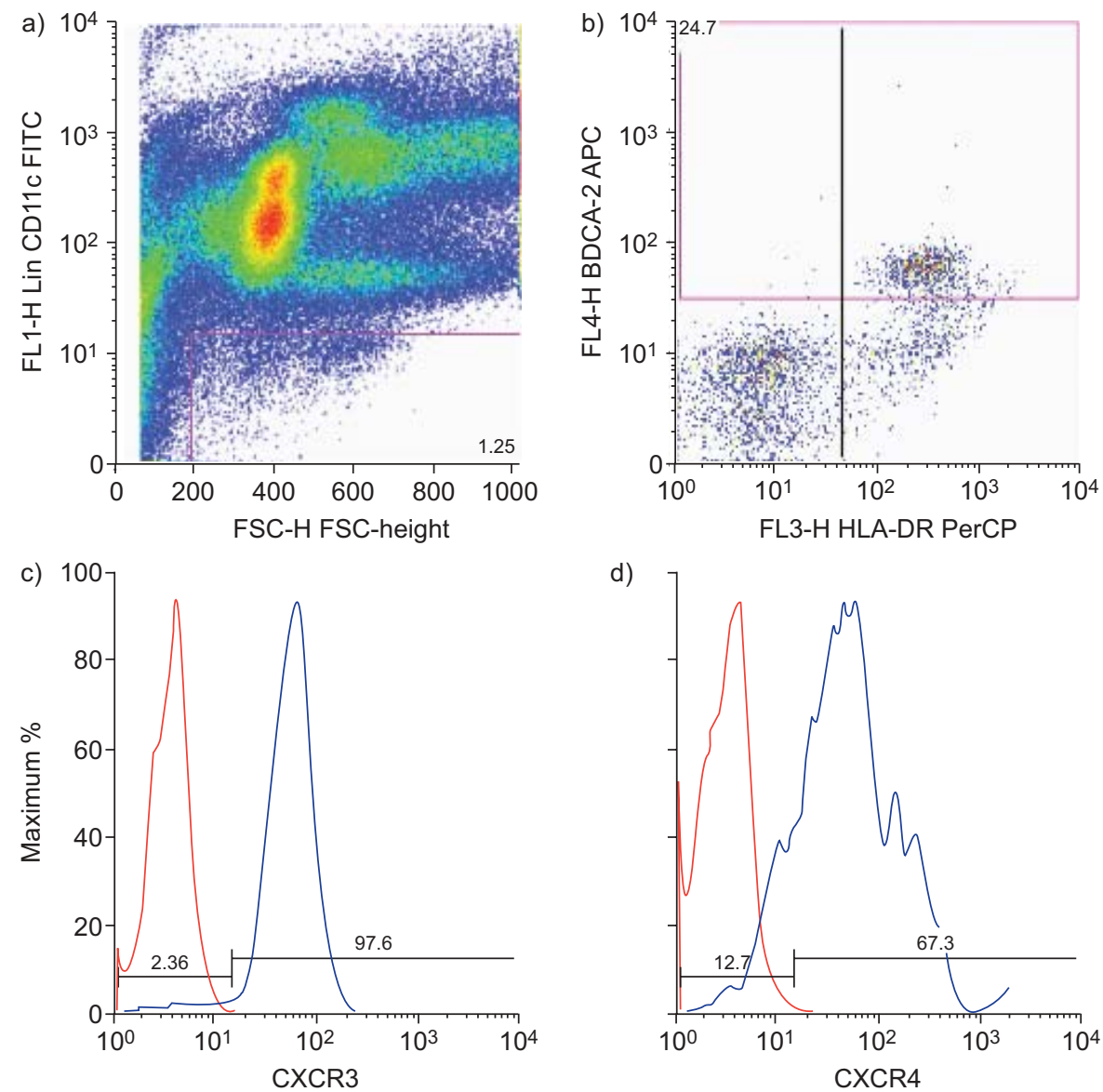

d)
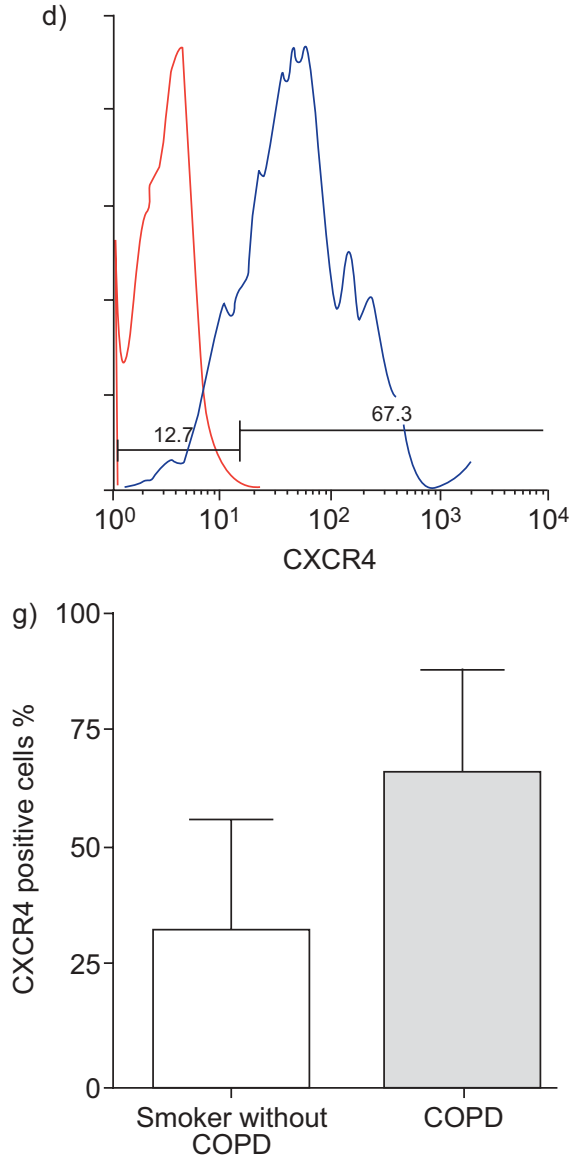

e)
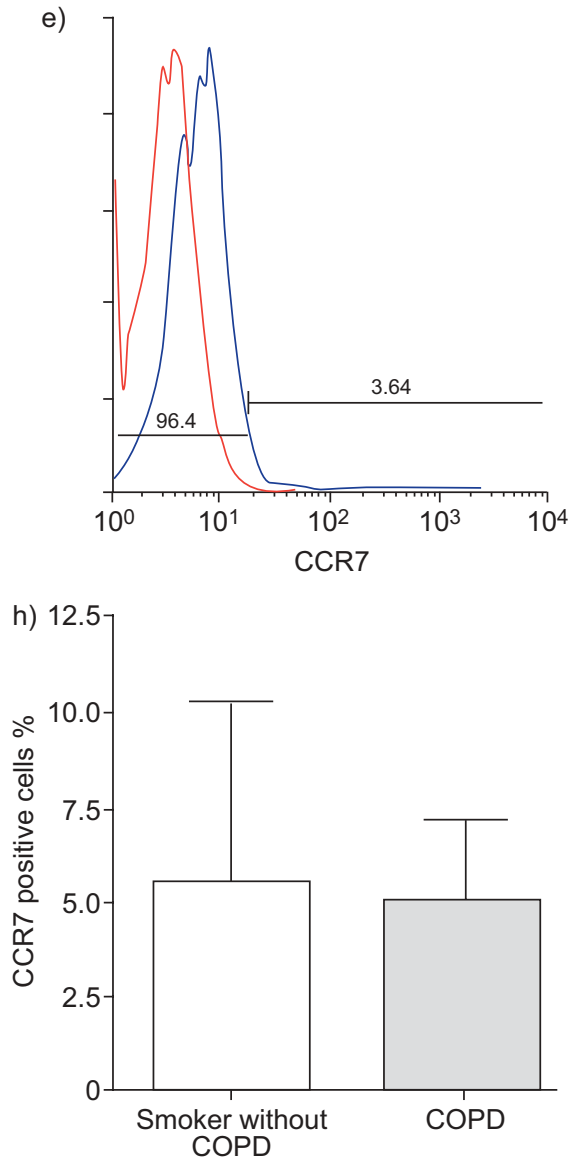

FIGURE 2. Chemokine receptor expression on pulmonary plasmacytoid dendritic cells (pDCs). Pulmonary pDCs were identified by flow cytometry on single cell suspensions of enzymatically digested human lung tissue. a) and b) Gating strategy for identification of pDCs: first, lineage negative, CD11c negative and low autofluorescent cells were selected (a), then gating focused on HLA-DR and BDCA-2 double positive cells (b). Within these pDCs, expression of lymphoid homing chemokine receptors c) CXCR3, d) CXCR4 and e) CCR7 was evaluated (blue histograms). Red histograms represent isotype control staining. Data are from a patient with chronic obstructive pulmonary disease (COPD). $\mathrm{f}-\mathrm{h}$ Comparison of the chemokine receptor expression on pulmonary pDC between smokers without COPD ( $\mathrm{n}=3$ ) and patients with COPD $(\mathrm{n}=2)$. Data are represented as mean \pm SEM. f) CXCR3 expression in $\mathrm{PDC}, \mathrm{g}) \mathrm{CXCR4}$ expression on $\mathrm{PDC}$ and h) CCR7 expression on pDC.

$(\mathrm{p}=0.02)$, and tended to be higher in COPD GOLD stage III-IV compared to never smokers $(p=0.06)$ (fig. $3 b)$.

The number of pDCs was significantly higher in lymphoid follicles of mild to moderate COPD patients compared with smokers without airflow limitation and compared with patients with severe to very severe COPD $(\mathrm{p}=0.004$ and $\mathrm{p}=0.04$, respectively) (fig. $3 \mathrm{c}$ ). There were no significant differences between never-smokers and smokers without COPD. Importantly, when pDCs were quantified in follicles with a confirmed B-cell zone in the adjacent section (which constitute $30 \%$ of all follicles), a similar trend towards 
TABLE 1 Characteristics of study population (immunohistochemical study)

\begin{tabular}{|c|c|c|c|c|c|}
\hline & Never-smokers & $\begin{array}{l}\text { Smokers without } \\
\text { COPD }\end{array}$ & COPD I-II & COPD III-IV & p-value ${ }^{\#}$ \\
\hline Subjects & 10 & 22 & 28 & 14 & \\
\hline Sex ratio male/female & $3 / 7$ & $17 / 5$ & $27 / 1$ & $6 / 8$ & $\mathrm{p}<0.001$ \\
\hline Age yrs & $56.3 \pm 11.2$ & $59.9 \pm 10.6$ & $63.3 \pm 9.0$ & $58.8 \pm 5.4$ & $\mathrm{p}=0.17$ (NS) \\
\hline Smoking history pack-yrs & 0 & $33.0 \pm 26.2$ & $45.9 \pm 22.4$ & $30.7 \pm 16.0$ & $p<0.001$ \\
\hline Smoking status current/ex-smoker & NA & $12 / 10$ & $19 / 9$ & $3 / 11$ & $p=0.02$ \\
\hline FEV $1 \%$ pred & $100.6 \pm 12.1$ & $103.2 \pm 17.8$ & $80.9 \pm 11.0$ & $29.9 \pm 10.4$ & $p<0.001$ \\
\hline FEV1/FVC \% & $77.3 \pm 7.3$ & $77.8 \pm 5.2$ & $60.1 \pm 6.0$ & $40.4 \pm 11.6$ & $\mathrm{p}<0.001$ \\
\hline LABA yes/no & $0 / 10$ & 0/22 & 2/26 & $12 / 2$ & $\mathrm{p}<0.001$ \\
\hline Inhaled corticosteroids yes/no & $0 / 10$ & $0 / 22$ & $0 / 28$ & $12 / 2$ & $p<0.001$ \\
\hline Inhaled corticosteroid dose $\mu$ g BDP per $24 \mathrm{~h}$ & 0 & 0 & 0 & $1353.9 \pm 659.1$ & $p<0.001$ \\
\hline Systemic corticosteroids yes/no & $0 / 10$ & $0 / 22$ & $0 / 28$ & $9 / 5$ & $p<0.001$ \\
\hline Systemic corticosteroid dose $\mathrm{mg}$ prednisolone per $24 \mathrm{~h}$ & 0 & 0 & 0 & $4.6 \pm 4.1$ & $p<0.001$ \\
\hline
\end{tabular}

accumulation of pDCs was observed in patients with mild to moderate COPD (figure E5 in the online supplementary material). Representative cryosections for the different study groups are shown in figure 4 .

\section{Quantification of pDCs in lung digests}

In a second independent study population, pDCs were quantified by flow cytometry. pDCs were identified in mononuclear single cell suspensions of human lung digests as BDCA-2- positive cells within the low autofluorescent, CD3negative, CD19-negative gate. The characteristics of the study population are shown in table 2 .

The number of pDCs in the single cell suspensions of COPD patients (GOLD stage I-II) was significantly higher compared with smokers without airflow limitation $(p=0.02)$ (fig. $3 d)$.

\section{CSE suppresses maturation-associated co-stimulatory molecule expression of $P D C$ in vitro}

Human Blood derived "untouched" pDCs were exposed to a range of concentrations of CSE to determine the effect on cellular viability. CSE concentrations from $2 \%$ had a detrimental effect on cellular viability, as shown in figure E6 in the online supplementary material.

Blood-derived pDCs were matured by adding imiquimod (a TLR-7 agonist) or CpG oligonucleotides (a TLR-9 agonist) in the presence or absence of CSE (0.5 or $1 \%$ ). TLR-7 and -9 stimulation was used to induce maturational response in CSEexposed pDCs. There was an attenuation of the maturational response in cigarette smoke exposed $\mathrm{pDCs}$ with a significantly impaired expression of CD83 in CpG/CSE-stimulated pDCs and of CD80, CD83 and CD86 in imiquimod/CSE-stimulated pDCs of healthy nonsmoking subjects. A similar reduced maturational response due to CSE was observed in pDCs of patients with COPD (fig. 5 and fig. E7 in the online supplementary material).

\section{CSE alters CpG-induced cytokine production by pDCs in vitro}

pDCs were stimulated with CpG oligonucleotides in the presence or absence of CSE (1\%) (fig. 6a, c, e). CSE attenuated the production of interferon- $\alpha$ by $\mathrm{CpG}$, both in healthy subjects and in patients with COPD. Remarkably, there was a significantly lower production of interferon- $\alpha$ by CSE-exposed pDCs of patients with COPD compared with pDC of healthy subjects. The production of tumour necrosis factor (TNF)- $\alpha$ and interleukin (IL)-8 already tended to be higher at baseline in pDCs of patients with COPD. The CpG-induced production of IL- 8 and TNF- $\alpha$ was increased by CSE in healthy subjects, but was not significantly altered by CSE in patients with COPD.

\section{CSE alters imiquimod-induced cytokine production by pDCs in vitro}

In imiquimod-stimulated pDCs, CSE induced a marked decrease in TNF- $\alpha$ production in both pDCs from healthy subjects, and in pDCs from patients with COPD (fig. 6b, d, f). We observed significantly higher levels of TNF- $\alpha$ and IL- 8 produced by $\mathrm{pDC}$ of patients with COPD compared to $\mathrm{pDCs}$ of healthy subjects.

There were no differences in production of IL-6, IL-10, IL-12 or IL-1 $\beta$ in CpG or imiquimod treated pDCs under the influence of $1 \%$ CSE (data not shown).

\section{DISCUSSION}

This study identified for the first time the presence of pDCs in small airways of human lungs and highlighted their presence in lymphoid follicles, a finding compatible with the expression of lymphoid homing chemokine receptors CXCR3 and CXCR4 on these cells. In addition, we showed a significant accumulation of pDCs in lymphoid follicles and lung digests of patients with mild to moderate COPD. We also demonstrated a marked influence of CSE on $\mathrm{pDC}$ function in vitro and showed that pDCs of patients with COPD are capable of producing higher levels of TNF- $\alpha$ and IL- 8 . 

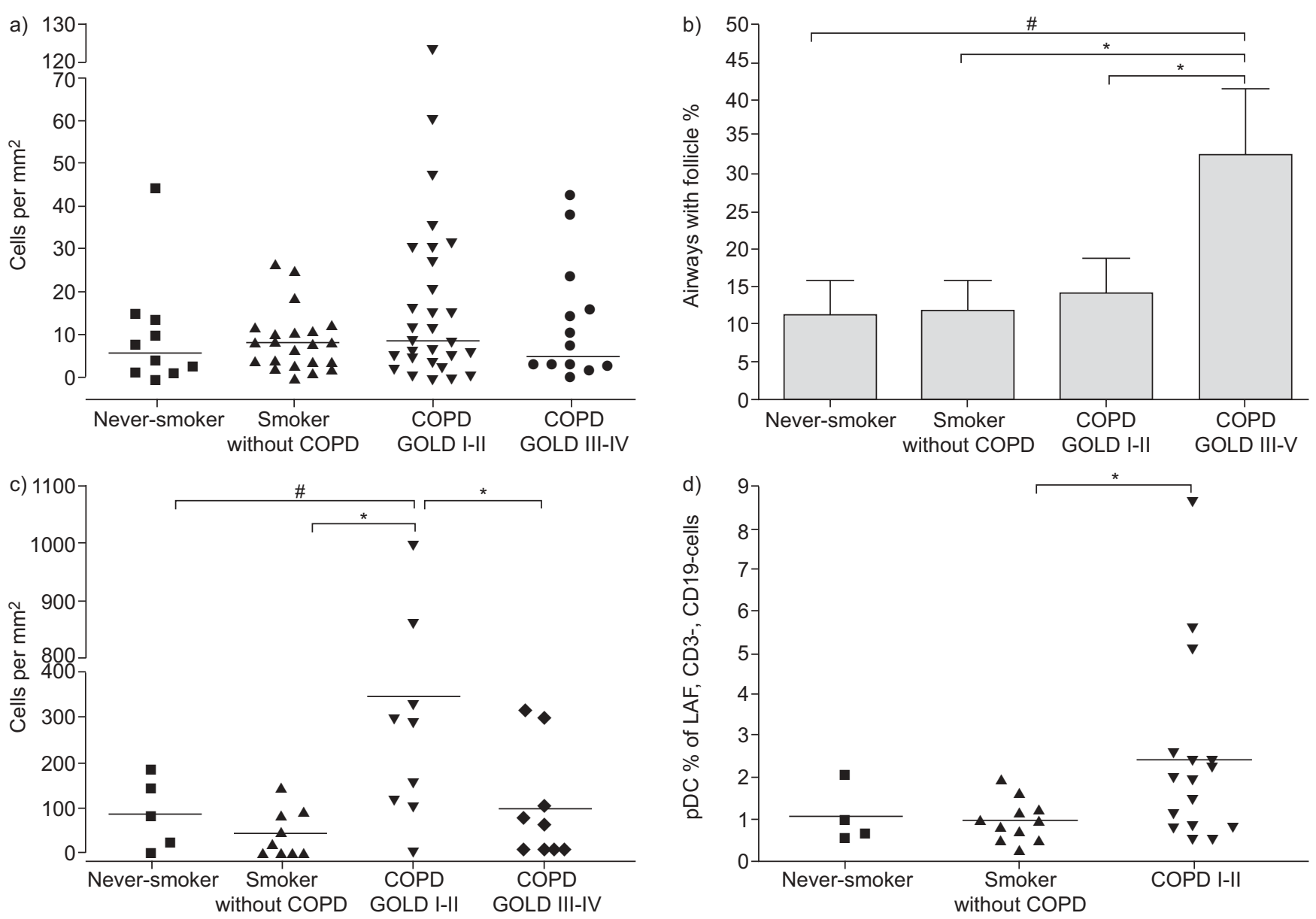

FIGURE 3. Quantification of plasmacytoid dendritic cells (pDCs) in small airways, in small airway-associated lymphoid follicles and in human lung digests a) BDCA-2positive pDCs, identified by immunohistochemical staining, were quantified in the small airway wall (excluding the area of lymphoid follicles) of never-smokers, smokers without chronic pulmonary obstructive disease (COPD), COPD Global Initiative for Chronic Obstructive Lung Disease (GOLD) stage I-II and COPD GOLD stage III-IV. Horizontal lines represent the median for each group. b) Quantification of lymphoid follicles. The mean \pm SEM of the percentage of small airways containing a lymphoid follicle is shown per group. *: significant differences $(p<0.05)$ between groups; ${ }^{*}: p=0.09$. c) BDCA-2- positive pDCs, identified by immunohistochemical staining, were quantified in the lymphoid follicles of never-smokers, smokers without COPD, COPD GOLD stage I-II and COPD GOLD stage III-IV. *: significant differences between groups ( $\mathrm{p}<0.05$ ); $\#: p=0.08$. d) pDCs were quantified by flow cytometry in mononuclear single cell suspensions of digested human lung tissue in an independent study population. pDC were identified as BDCA-2- positive cells. Quantification is expressed as the percentage of the low autofluorescent (LAF), CD3-negative, CD19-negative population. Horizontal lines indicate mean values. *: significant $p$-value $(p<0.05)$.

Although the presence of pDCs in human lungs is well established by studies using single cell suspensions of digested lung tissue or by analysing BAL fluid, the exact distribution and localisation of pDCs in small airways was unknown until now. In the past, MASTEN et al. [13] used immunohistochemical staining for CD123 on human lung tissue to identify pDCs, but this marker is nonspecific as it is also expressed by other cells such as mDCs, macrophages and granulocytes. Using the specific marker BDCA-2, pDCs were identified in low numbers in the mucosal surfaces of the small airways and were often found in lymphoid follicles. This predominant lymphoid localisation is parallel to the described distribution of pDCs in other peripheral tissues, such as nasal mucosa and synovium [24-26]. Indeed, blood pDCs are known to home directly from the blood circulation towards these lymphoid tissues via high endothelial venules under the influence of CXCR3 and CXCR4 ligands. Interestingly, a limited number of
pDCs was found outside these mucosa-associated lymphoid tissue structures in both individuals with and without COPD, indicating that $\mathrm{pDCs}$ can also migrate to peripheral nonlymphoid tissue.

Pulmonary BDCA-2-positive pDCs are mainly considered immature as shown previously on flow cytometric analysis of single cell suspensions of digested human lung tissue [12]. Upon maturation, $\mathrm{pDC}$ s downregulate BDCA-2, but retain the expression of CD123 [27]. In addition, our data show a low expression of the chemokine receptor CCR7, which is generally upregulated by DC during their maturation process. FREEMAN et al. [17] showed recently that BDCA-2-positive pDCs can express higher levels of maturation markers, especially in COPD. This suggests that the BDCA-2-positive cell population consists of immature and maturing $\mathrm{pDCs}$, whereas mature pDCs could become BDCA-2 negative. 

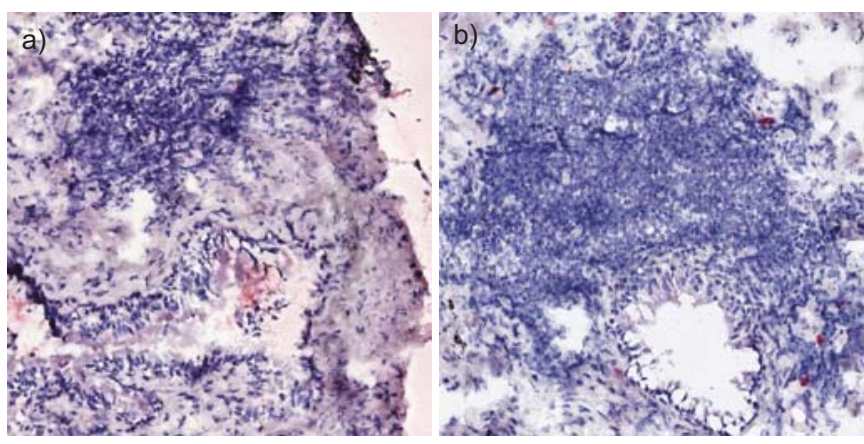

c).
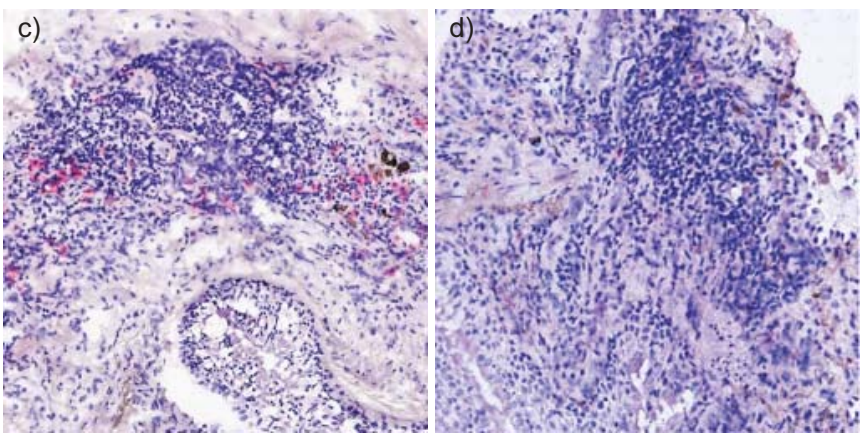

FIGURE 4. Plasmacytoid dendritic cells in lymphoid follicles. Immunohistochemical staining for blood dendritic cell antigen 2 on cryosections of human lungs, focusing on lymphoid follicles. a) Never-smoker, b) smoker without chronic obstructive pulmonary disease (COPD), c) COPD Global Initiative for Chronic Obstructive Lung Disease (GOLD) stage I, and d) COPD GOLD stage IV. Original magnification 200x

When quantifying the number of pDCs in the small airways of patients with COPD and subjects without airflow limitation, we found a significant accumulation of these cells in lymphoid follicles of patients with mild and moderate COPD compared with smokers without COPD. Importantly, these findings were confirmed by the flow cytometric quantification of pDCs in human lung digests of an independent study population. As lymphoid follicles harbour the majority of pDCs in the lungs, it is conceivable that the results of the flow cytometric study of lung
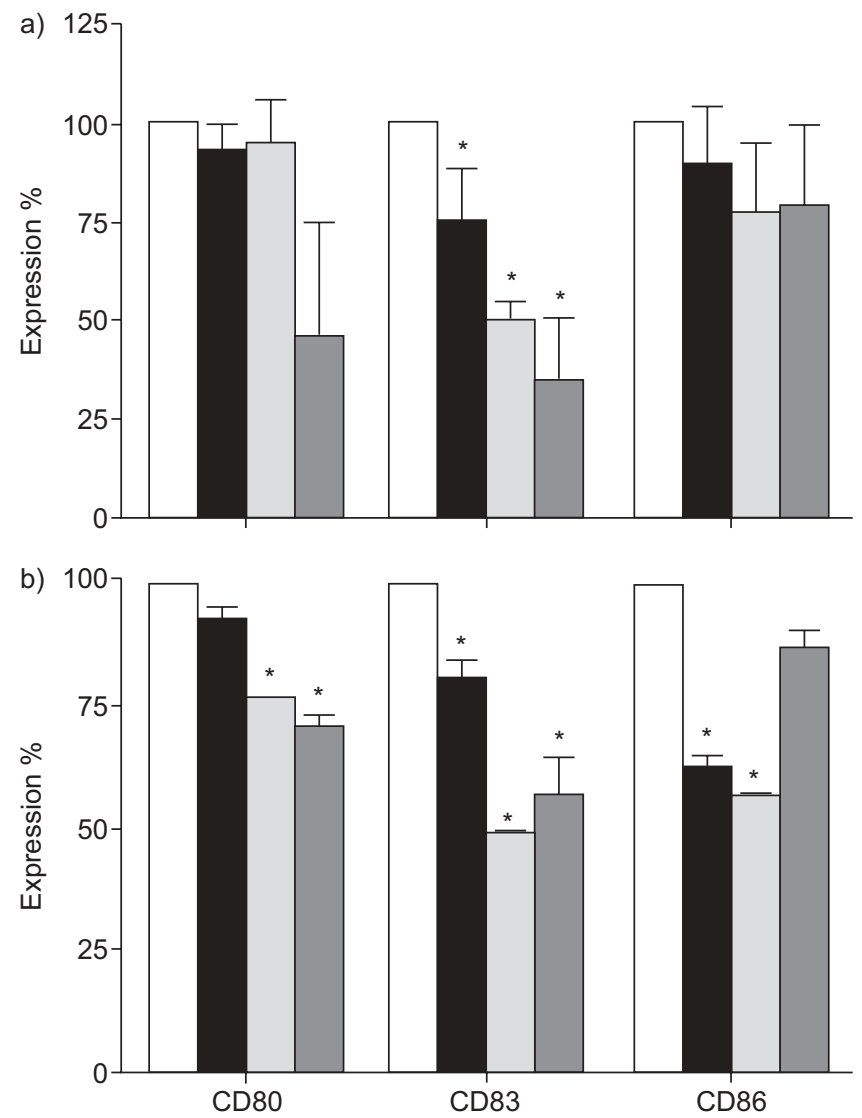

FIGURE 5. Cigarette smoke extract (CSE) impairs plasmacytoid dendritic cell (pDC) maturation. Blood-derived pDCs were exposed for $18 \mathrm{~h}$ to CSE and a maturation stimulus. Expression of maturation markers on viable pDCs was evaluated by flow cytometry. Mean \pm SEM upregulation of the maturation marker in $0.5 \%$ CSE ( $)$ and 1\% CSE ( $\square$ ) environment is depicted relative to the maturation response in absence of CSE. $\square$ : the relative maturation of $\mathrm{CpG}$ or imiquimod (IMQ) stimulated pDC of patients with chronic obstructive pulmonary disease in a 1\% CSE environment; $\square$ : control with $0 \%$ CSE. a) With CpG oligonucleotides as maturation stimulus; b) with IMQ as maturation stimulus. ${ }^{*}: p<0.05$. Data are representative for six and five independent experiments, respectively.

TABLE 2 Characteristics of study population (flow cytometric study)

\begin{tabular}{|c|c|c|c|c|}
\hline & Never-smokers & Smokers without COPD & COPD I-II & p-value ${ }^{\#}$ \\
\hline Subjects & 4 & 11 & 16 & \\
\hline Age yrs & $55.8 \pm 13.7$ & $62.4 \pm 8.9$ & $66.8 \pm 9.3$ & NS \\
\hline Smoking history pack-yrs & 0 & $34.2 \pm 15.7$ & $37.2 \pm 20.5$ & $p=0.007$ \\
\hline Smoking status current/ex-smokers & NA & $6 / 5$ & $8 / 8$ & NS \\
\hline LABA yes/no & $0 / 4$ & $0 / 11$ & $4 / 12$ & NS \\
\hline Inhaled corticosteroids yes/no & $0 / 4$ & $0 / 11$ & $1 / 15$ & NS \\
\hline Systemic corticosteroids yes/no & $0 / 4$ & $0 / 11$ & $0 / 16$ & NS \\
\hline
\end{tabular}

Data are expressed as mean \pm SD or $\mathrm{n}$, unless otherwise stated. COPD: chronic obstructive pulmonary disease; FEV 1 : forced expiratory volume in $1 \mathrm{~s}$; \% pred: \% predicted FVC: forced vital capacity; LABA: long-acting $\beta_{2}$ - agonist; NA: not available; NS: nonsignificant. ${ }^{*}$ : generated by Kruskal-Wallis test for continuous variables and Fisher exact test for categorical variables. 

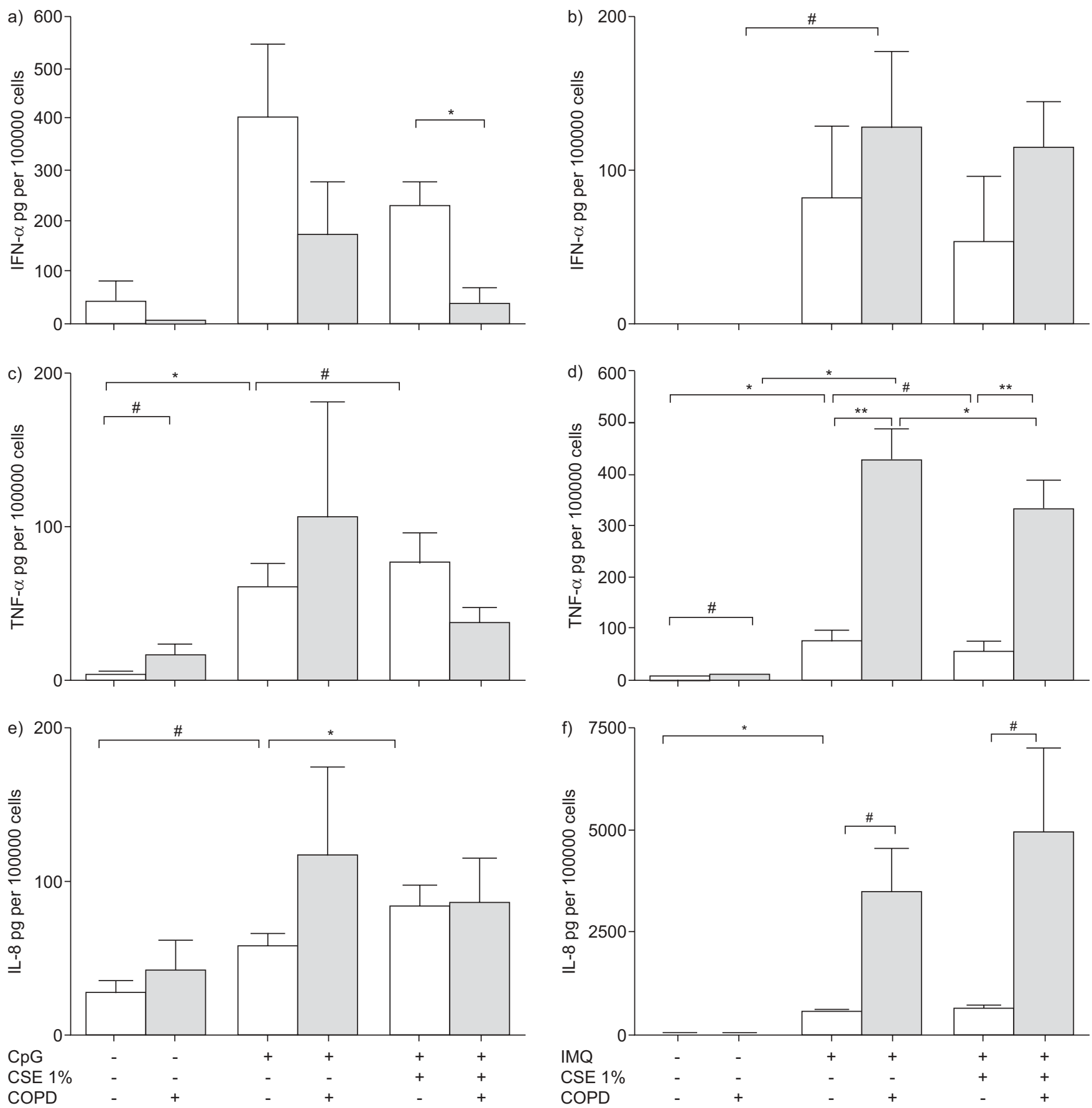

FIGURE 6. Cigarette smoke extract (CSE) alters cytokine production of plasmacytoid dendritic cells (pDCs) in response to CpG oligonucleotides or imiquimod (IMQ). Blood pDCs of healthy subjects and patients with chronic obstructure pulmanary disease (COPD) were incubated for $18 \mathrm{~h}$ with or without CpG oligonucleotides or IMQ in the presence or absence of CSE (1\%). a, b) Interferon (IFN)- $\alpha$, c, d) tumour necrosis factor (TNF)- $\alpha$ and e, f) interleukin (IL)-8 concentrations were measured in the culture supernatant. Data are presented as mean \pm SEM. ${ }^{*}: p<0.05 ;{ }^{*}: p<0.01 ;{ }^{*}: p-v a l u e s$ between $0.05-0.08$. Data represent six (for $\mathrm{CpG}$ ) and five (for IMQ) independent experiments.

digests are mirrored in the quantification of pDCs in lymphoid follicles. The accumulation of pDCs in lymphoid follicles is compatible with the observed increase of CXCR3 expression on pulmonary pDCs of patients with COPD in our study, and with the recently published observations of increased follicular expression of CXCR3 in patients with COPD [22].
In contrast, we found no differences in pDC numbers between never smokers and smokers without airflow limitation. These results are in accordance with the findings in BAL fluid after acute smoke exposure [28] and indicate that smoking as such does not influence the number of pDCs in the airways and lymphoid follicles. 
As immature pDCs are present in the mucosal areas of the lung, they can be directly influenced by the effects of cigarette smoke. We therefore incubated pDCs with CSE, mimicking mainsteam cigarette smoke exposure. This resulted in a blunted maturation response of these cells when imitating viral infection by using the TLR-9 agonist CpG oligonucleotides or the TLR-7 agonist imiquimod. These findings are in accordance with the previously published observations of impaired maturation of CSE-exposed mDCs [23].

Moreover, we found an altered cytokine production during the maturation process with a reduction of the interferon- $\alpha$ response by CSE in TLR-9 stimulated pDCs. This blunted interferon- $\alpha$ response is both present in pDCs of healthy subjects and in pDCs of patients with COPD. This finding is important, as interferon- $\alpha$ production is the cardinal innate feature of pDCs and a cornerstone of early antiviral defence. A blunted type-I interferon response combined with impaired maturational capabilities of pDCs due to cigarette smoking could contribute to the increased susceptibility of smokers to viral infections [29] and the development of low grade infections in patients with COPD, stimulating the ongoing pathogenic inflammatory process [30]. In addition, CSE induced an increase of IL-8 production in maturing pDCs of healthy subjects, which could contribute to the increased influx of neutrophils in smokers. In maturing pDCs of patients with COPD, a significantly higher production of IL- 8 and TNF- $\alpha$ is present compared with maturing pDCs of healthy subjects. In the case of IL-8, CSE cannot further augment this production, whereas CSE decreases the production of TNF- $\alpha$ in pDC of patients with COPD. As increased maturation status of pDCs has been described in COPD [17], this enhanced production of TNF- $\alpha$ and IL- 8 by pDCs could also be present in vivo, contributing to the inflammatory process in COPD.

Importantly, our in vitro data confirm and extend the recently published in vitro study by MORTAZ et al.[31] that demonstrated impaired interferon- $\alpha$ response and increased IL- 8 production in CPG stimulated CSE exposed pDC of healthy subjects.

The exact role of the accumulating pDCs in lymphoid follicles of patients with mild to moderate COPD remains to be elucidated. One could speculate that this increased number of pDCs reflects an influx of these cells as a compensatory mechanism which tries to dampen the enhanced immune response in COPD, as pDCs are known for their tolerogenic properties. Accordingly, a recent publication showed an accumulation of regulatory CD4+Foxp3+ T-cells in lymphoid follicles of patients with moderate COPD compared with smokers and nonsmokers without COPD [32]. pDCs could also play an important role in lymphoid neogenesis and the homeostasis of tertiary lymphoid structures in the lung $[33,34]$. In addition to the increased influx of pDCs, a reduced DC maturational process in COPD, as suggested by TsOUMAKIDOU et al. [19], could contribute to the accumulation of immature BDCA-2-positive DCs in lymphoid follicles of patients with COPD.

Importantly, in the most severe stages of COPD, the number of BDCA-2-positive DCs was at the same level as in the individuals without airflow limitation. This could reflect a relative depletion of $\mathrm{pDCs}$, as often seen in chronic low grade viral infections [35]. An increased maturational response of
pDCs in severe COPD, as suggested by FREEMAN et al. [17], could also explain the lower numbers of BDCA-2-positive pDCs. Finally, the use of inhaled and systemic corticosteroids by patients with severe and very severe COPD could contribute to the reduced number of pDCs in this study, as these drugs are known to induce apoptosis in circulating and tissue-resident pDCs [36-39]. This lower number of pDCs in lymphoid follicles of patients with very severe COPD treated with inhaled steroids could be linked to the observed association of increased risk of pneumonia and the use of inhaled corticosteroids in patients with COPD [40].

A reduction in pDC numbers could lead to a state of "loss of tolerance", which can be linked to a drop in regulatory T-cell numbers and the occurrence of autoimmune processes in the most severe stages of COPD [41]. In addition, an imbalance between $\mathrm{mDC}$ and $\mathrm{pDCs}$ in the lungs could cause a derailment in the control of inflammation. Indeed, in COPD GOLD stage I, we observed a significant positive correlation between the number of langerin-positive mDCs and the number of $\mathrm{pDCs}$ in the total airway wall. However, this positive correlation was completely lost in COPD GOLD stage III-IV. This suggests that langerin-positive mDCs (accumulating in small airways of patients with COPD and stimulating T-helper 1 and T-helper 17 responses) dominate over the tolerogenic pDC [20].

There are several aspects that strengthen this study. The immunohistochemical study addressed for the first time the number of $\mathrm{pDCs}$ in human lungs of a large population, using a specific pDC marker, covering the different severities of COPD and comparing them to individuals without airflow limitation. Descriptive quantitative ex vivo data were supplemented with in vitro functional testing, highlighting the influence of CSE on pDC function.

There are, however, several limitations which should be addressed. First, tissue samples for the immunohistochemical and flow cytometric studies were mainly obtained from patients operated for a lung tumour. Theoretically, this tumour could influence the number and phenotype of the pDCs. However, tissue samples were obtained at a maximal distance from this lesion, minimising its effect.

Secondly, quantification of immunohistochemical stainings for pDCs in small airways and follicles revealed rather low numbers of these cells. This might have an impact on the sensitivity for detecting differences in pDC numbers between study groups. However, the immunohistochemical study data were confirmed by flow cytometric quantification of pDCs in an independent population. In addition, our data are in line with a recent flow cytometric study on lung digests by FREEMAN et al [17], showing a tendency towards higher pDC numbers in patients with moderate COPD.

Finally, the in vitro experiments with CSE only investigated the direct influence of smoke on the pDC. However, the indirect effect of cigarette smoke (through mediators released by smokeexposed epithelium, inflammatory and structural cells) can greatly influence the function of these antigen-presenting cells.

\section{Conclusions}

Cigarette smoking does not influence the number of pDCs in human lungs. However, pDCs are functionally altered by CSE, 
resulting in impaired antiviral defence mechanisms. This observation directly links pDC function to the increased susceptibility of smokers to viral infections.

pDCs are present in the small airways of healthy subjects and patients with COPD. In COPD, maturing pDCs can produce higher levels of TNF- $\alpha$ and IL-8, contributing to enhanced influx of neutrophils and the activation of macrophages in the lungs of patients with COPD.

Finally, pulmonary pDCs, through the enhanced expression of the lymphoid homing chemokine receptor CXCR3, accumulate significantly in lymphoid follicles of patients with mild and/or moderate COPD. However, in the most severe stages of COPD, the number of pDCs is reduced, which could be compatible with an impaired control on the adaptive immune response and a loss of tolerance in end-stage COPD.

\section{SUPPORT STATEMENT}

This work was supported by the Fund for Scientific Research in Flanders (FWO Vlaanderen, research projects G.0011.03 and G0343.01N), by Project grant 01G01009 from the Concerted Research Initiative of the Ghent University and by the Interuniversity Attraction Poles programme (IUAP) Belgian state -Belgian Science Policy P6/35. G.R. Van Pottelberge is a doctoral research fellow of the Fund for Scientific Research in Flanders. K.R. Bracke is a postdoctoral researcher of the Fund for Scientific Research in Flanders.

\section{STATEMENT OF INTEREST}

Statements of interest for G.R. van Pottelberge and G.G. Brusselle can be found at www.erj.ersjournals.com $/ \mathrm{misc} /$ statements.dtl

\section{ACKNOWLEDGEMENTS}

The authors thank A. Neesen (Dept of Respiratory Medicine, Ghent University Hospital, Ghent, Belgium), I. De Borle (Dept of Respiratory Medicine, Ghent University Hospital), K. De Saedeleer (Dept of Respiratory Medicine, Ghent University Hospital), E. Castrique (Dept of Respiratory Medicine, Ghent University Hospital), A. Goethals (Dept of Respiratory Medicine, Ghent University Hospital), E.J.J. Vulto-De Groot (Dept of Otolaryngology of the Academic Medical Center, Amsterdam, the Netherlands), E.J. van der Toom (Dept of Otolaryngology of the Academic Medical Center), D. Van Egmond (Dept of Otolaryngology of the Academic Medical Center), S. Verschraegen (Dept of Respiratory Medicine, Ghent University Hospital) and L. Raman (Dept of Respiratory Medicine, Ghent University Hospital) for their technical contribution to this work. The authors would also like to thank M. Hamels (Dept of Internal Medicine, Ghent University Hospital) for carefully reading the manuscript, D. Testelmans (Dept of Respiratory Medicine, University Hospital Gasthuisberg, Leuven, Belgium), J Vernooy (Nutrim, Dept Of Respiratory Medicine, University of Maastricht, Maastricht, the Netherlands) and M Dentener (Nutrim, Dept of Respiratory Medicine, University of Maastricht, Maastricht, the Netherlands) for providing the patient characteristics of the GOLD stage IV patients. The authors would like to thank H. Middendorp (Dept of Respiratory Medicine, Ghent University Hospital), F. Vandewalle (Dept of Respiratory Medicine, Ghent University Hospital) and T. Verstraete (Dept of Respiratory Medicine, Ghent University Hospital) for their support in data management.

\section{REFERENCES}

1 Lopez AD, Mathers CD. Measuring the global burden of disease and epidemiological transitions: 2002-2030. Ann Trop Med Parasitol 2006; 100: 481-499.

2 Rabe KF, Hurd S, Anzueto A, et al. Global strategy for the diagnosis, management, and prevention of chronic obstructive pulmonary disease: GOLD executive summary. Am J Respir Crit Care Med 2007; 176: 532-555.

3 Curtis JL, Freeman CM, Hogg JC. The immunopathogenesis of chronic obstructive pulmonary disease: insights from recent research. Proc Am Thorac Soc 2007; 4: 512-521.

4 Chung KF, Adcock IM. Multifaceted mechanisms in COPD: inflammation, immunity, and tissue repair and destruction. Eur Respir J 2008; 31: 1334-1356.

5 Hogg JC, Chu F, Utokaparch S, et al. The nature of small-airway obstruction in chronic obstructive pulmonary disease. $N$ Engl $J$ Med 2004; 350: 2645-2653.

6 Steinman RM, Banchereau J. Taking dendritic cells into medicine Nature 2007; 449: 419-426.

7 Vermaelen K, Pauwels R. Pulmonary dendritic cells. Am J Respir Crit Care Med 2005; 172: 530-551.

8 Schettini J, Mukherjee P. Physiological role of plasmacytoid dendritic cells and their potential use in cancer immunity. Clin Dev Immunol 2008; 2008: 106321.

9 Demedts IK, Bracke KR, Maes T, et al. Different roles for human lung dendritic cell subsets in pulmonary immune defense mechanisms. Am J Respir Cell Mol Biol 2006; 35: 387-393.

10 Puccetti P, Fallarino F. Generation of T cell regulatory activity by plasmacytoid dendritic cells and tryptophan catabolism. Blood Cells Mol Dis 2008; 40: 101-105.

11 Ito $\mathrm{T}$, Yang M, Wang $\mathrm{YH}$, et al. Plasmacytoid dendritic cells prime IL-10-producing $\mathrm{T}$ regulatory cells by inducible costimulator ligand. J Exp Med 2007; 204: 105-115.

12 Demedts IK, Brusselle GG, Vermaelen KY, et al. Identification and characterization of human pulmonary dendritic cells. Am J Respir Cell Mol Biol 2005; 32: 177-184.

13 Masten BJ, Olson GK, Tarleton CA, et al. Characterization of myeloid and plasmacytoid dendritic cells in human lung. J Immunol 2006; 177: 7784-7793.

14 Tsoumakidou M, Tzanakis N, Papadaki HA, et al. Isolation of myeloid and plasmacytoid dendritic cells from human bronchoalveolar lavage fluid. Immunol Cell Biol 2006; 84: 267-273.

15 Bratke K, Lommatzsch M, Julius P, et al. Dendritic cell subsets in human bronchoalveolar lavage fluid after segmental allergen challenge. Thorax 2007; 62: 168-175.

16 Demedts IK, Bracke KR, Van Pottelberge GR, et al. Accumulation of dendritic cells and increased CCL20 levels in the airways of patients with chronic obstructive pulmonary disease. Am J Respir Crit Care Med 2007; 175: 998-1005.

17 Freeman CM, Martinez FJ, Han MK, et al. Lung dendritic cell expression of maturation molecules increases with worsening chronic obstructive pulmonary disease. Am J Respir Crit Care Med 2009; 180: 1179-1188.

18 Lommatzsch M, Bratke K, Bier A, et al. Airway dendritic cell phenotypes in inflammatory diseases of the human lung. Eur Respir J 2007; 30: 878-886.

19 Tsoumakidou M, Koutsopoulos AV, Tzanakis N, et al. Decreased small airway and alveolar CD83+ dendritic cells in COPD. Chest 2009; 136: 726-733.

20 Van Pottelberge GR, Bracke KR, Joos GF, et al. The role of dendritic cells in the pathogenesis of COPD: Liaison Officers in the Front line. COPD 2009; 6: 284-290.

21 Saetta M, Di Stefano A, Turato G, et al. CD8+ T-lymphocytes in peripheral airways of smokers with chronic obstructive pulmonary disease. Am J Respir Crit Care Med 1998; 157: 822-826.

22 Kelsen SG, Aksoy MO, Georgy M, et al. Lymphoid follicle cells in chronic obstructive pulmonary disease overexpress the chemokine receptor CXCR3. Am J Respir Crit Care Med 2009; 179: 799-805.

23 Vassallo R, Tamada K, Lau JS, et al. Cigarette smoke extract suppresses human dendritic cell function leading to preferential induction of Th-2 priming. J Immunol 2005; 175: 2684-2691.

24 Lande R, Giacomini E, Serafini B, et al. Characterization and recruitment of plasmacytoid dendritic cells in synovial fluid and 
tissue of patients with chronic inflammatory arthritis. J Immunol 2004; 173: 2815-2824.

25 Bangert C, Friedl J, Stary G, et al. Immunopathologic features of allergic contact dermatitis in humans: participation of plasmacytoid dendritic cells in the pathogenesis of the disease? J Invest Dermatol 2003; 121: 1409-1418.

26 Jahnsen FL, Farkas L, Lund-Johansen F, et al. Involvement of plasmacytoid dendritic cells in human diseases. Hum Immunol 2002; 63: 1201-1205.

27 Dzionek A, Fuchs A, Schmidt P, et al. BDCA-2, BDCA-3, and BDCA-4: three markers for distinct subsets of dendritic cells in human peripheral blood. J Immunol 2000; 165: 6037-6046.

28 Lommatzsch M, Bratke K, Knappe T, et al. Acute effects of tobacco smoke on human airway dendritic cells in vivo. Eur Respir J 2010; 35: 1130-1136.

29 Arcavi L, Benowitz NL. Cigarette smoking and infection. Arch Intern Med 2004; 164: 2206-2216.

30 Sethi S, Murphy TF. Infection in the pathogenesis and course of chronic obstructive pulmonary disease. $N$ Engl J Med 2008; 359: 2355-2365.

31 Mortaz E, Lazar Z, Koenderman L, et al. Cigarette smoke attenuates the production of cytokines by human plasmacytoid dendritic cells and enhances the release of IL-8 in response to TLR-9 stimulation. Respir Res 2009; 10: 47.

32 Plumb J, Smyth LJ, Adams HR, et al. Increased T-regulatory cells within lymphocyte follicles in moderate COPD. Eur Respir J 2009; 34: 89-94.
33 Brusselle GG, Demoor T, Bracke KR, et al. Lymphoid follicles in (very) severe COPD: beneficial or harmful? Eur Respir J 2009; 34: 219-230.

34 Aloisi F, Pujol-Borrell R. Lymphoid neogenesis in chronic inflammatory diseases. Nat Rev Immunol 2006; 6: 205-217.

35 Retamales I, Elliott WM, Meshi B, et al. Amplification of inflammation in emphysema and its association with latent adenoviral infection. Am J Respir Crit Care Med 2001; 164: 469-473.

36 Shodell M, Shah K, Siegal FP. Circulating human plasmacytoid dendritic cells are highly sensitive to corticosteroid administration. Lupus 2003; 12: 222-230.

37 Abe M, Thomson AW. Dexamethasone preferentially suppresses plasmacytoid dendritic cell differentiation and enhances their apoptotic death. Clin Immunol 2006; 118: 300-306.

38 Hoetzenecker W, Meindl S, Stuetz A, et al. Both pimecrolimus and corticosteroids deplete plasmacytoid dendritic cells in patients with atopic dermatitis. I Invest Dermatol 2006; 126: 2141-2144.

39 Boor PP, Metselaar HJ, Mancham S, et al. Prednisolone suppresses the function and promotes apoptosis of plasmacytoid dendritic cells. Am J Transplant 2006; 6: 2332-2341.

40 Ernst P, Gonzalez AV, Brassard P, et al. Inhaled corticosteroid use in chronic obstructive pulmonary disease and the risk of hospitalization for pneumonia. Am J Respir Crit Care Med 2007; 176: 162-166.

41 Cosio MG, Saetta M, Agusti A. Immunologic aspects of chronic obstructive pulmonary disease. N Engl J Med 2009; 360: 2445-2454. 